\title{
La perfusión como técnica de practica avanzada en enfermería
}

\section{Hemoperfusion as an Advanced Practice Technique in Nursing}

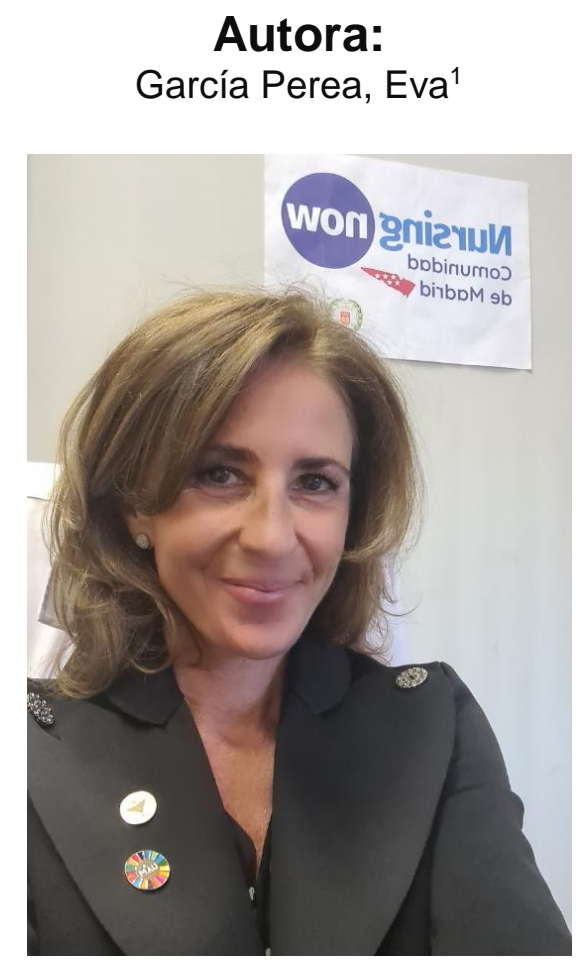

1. Directora del Departamento de Enfermería, Facultad de Medicina. Representante de la CIT de la CNDE. Representante de Universidades Nursing Now Comunidad de Madrid. Universidad autónoma de Madrid. España. ORCID: http://orcid.org/0000-0002-6972-3261. Correo electrónico: eva.garcia@uam.es

Palabras clave: hemoperfusion; enfermería; practica avanzada.

Keywords: hemoperfusion; nursing; advanced practice.

Puede citar este artículo como: García Perea E. La perfusión como técnica de practica avanzada en enfermería. RECIEN. Revista Científica de Enfermería. 2021; 21: 1-3. https://doi.org/10.14198/recien.2021.21.01 
Durante los últimos años la práctica avanzada en enfermería, se va desarrollando progresivamente para dar respuesta al incremento de la complejidad de los pacientes y de la tecnología que, requieren cada vez una formación adicional a la que se recibe durante la formación de Grado. Un ejemplo de practica avanzada, es la figura de enfermería en los procesos que afectan a pacientes con problemas circulatorios y requieren un manejo adecuado de las técnicas de hemoperfusión.

En la Facultad de Medicina de la Universidad Autónoma de Madrid y desde el Departamento de Enfermería, se ha implementado para el presente curso 2021-2022 un Máster (título propio) de Práctica Clínica Avanzada en Circulación Extracorpórea y Perfusión. Nos ha sorprendido la demanda de enfermeras y enfermeros perfusionistas de todo el territorio nacional y para avanzar en el proceso de consolidación y mejora de esta formación. Para avanzar, la UAM y la Consejería de Sanidad de la Comunidad autónoma de Madrid, hemos firmado un convenio que hablita las prácticas clínicas de los estudiantes de este título en todos los hospitales de la Comunidad de Madrid donde se practiquen técnicas de circulación extracorpórea, de manera que se facilite la formación e incorporación de enfermeras y enfermeros de práctica avanzada en técnicas extracorpóreas y perfusión a puestos de trabajo muy perfilados. El programa cuenta con el apoyo de sociedades de tanto prestigio como CGE, CODEM y ANDE.

La dirección del master une dos disciplinas, medicina y enfermería tanto para la dirección del programa como la participación del resto de profesorado que colabora en el mismo.

Con gran ilusión nos proponemos formar a diplomados y graduados de enfermería que quieran aprender el manejo de las técnicas de circulación y oxigenación extracorpórea con las garantías de calidad y seguridad pertinentes tanto para el paciente que precise una cirugía en extracorpórea como para el paciente que requiere de este tratamiento en una Unidad de Cuidados Críticos. El programa incluye además un Diploma de especialización en práctica avanzada en atención integral al paciente en ECMO y/o portador de dispositivos de asistencia ventricular, especialmente diseñado para profesionales que ejercen en el ámbito de los Cuidados Intensivos. Es una formación de 100 ECTS con dos años de duración, siguiendo los patrones europeos para la formación avanzada y tiene carácter semipresencial, lo que facilita conjugar metodologías docentes adecuadas para el perfil del estudiante y los contenidos a impartir. Si hay profesionales interesados en consultar el contenido, pueden hacerlo por mail en el correo secretaria.formacioncontinua@fuam.es.

Esta iniciativa puede suponer un avance en el camino de la definición de enfermería de practica avanzada que sin duda se complementa con las propias especialidades regladas 
de enfermería y otros programas ofrecidos por distintas universidades y centros de formación de prestigio.

Desde la dirección del departamento de enfermería de la UAM proponemos abrir foros de debate para avanzar en un tema importante como es el perfil de enfermería de practica avanzada que seguramente beneficiara tanto a la profesión como a las personas que reciben nuestros cuidados. 